\title{
Piezoelectric thermoelastic dissipation research of piezoelectric harvester under different vibration
}

\author{
Yuting Liu', 2, a Jiahao Deng ${ }^{1,2}$, Yong Ye , 2, 3, Zhuo Hou $^{1,2,4}$, Zuodong Duan ${ }^{1,2}$ and Sha Xu1, 2 \\ ${ }^{1}$ School of Mechatronical Engineering, Beijing Institute of Technology, Beijing 100081, China \\ ${ }^{2}$ Science and Technology on Electromechanical Dynamic Control Laboratory, Beijing Institute of Technology, Beijing 100081, China \\ ${ }^{3}$ College of Information Engineering, Shenzhen University, Shenzhen 518060, China \\ ${ }^{4}$ National Key Laboratory of Electronic Measurement Technology, North University of China, Taiyuan 030051, China
}

\begin{abstract}
Piezoelectric materials are widely used to form piezoelectric energy harvesters. Also, the thermoelastic dissipation always influences the energy harvesting efficiency, during the energy harvest process. Therefore, in this paper, we discuss the effect of thermoelastic dissipation on the piezoelectric harvester through numerical calculation, simulation and experiment. The piezoelectric thermoelastic coupling governing equations under different vibration are derived, which are based on the Euler-Bernoulli beam theory, thermal conduction model and piezoelectric field model. Then, the structure frequency shift and thermoelastic damping are studied via numerical calculation and simulation. Meanwhile, we show the influence of the temperature field on the piezoelectric structure under different vibration modes. Furth more, we research the variations of piezoelectric structure thermoelastic dissipation characteristics under different structure geometry sizes. Based on these analyses, the effect of piezoelectric thermoelastic dissipation on the piezoelectric harvester is researched
\end{abstract}

\section{Introduction}

The piezoelectric material can be used to capture the external energy in the beam structure, which constitutes a piezoelectric harvester, completing the capture of energy. The key issues for the piezoelectric harvester include the design and manufacture of structures with high quality factor in the realization of its function. When the micro devices working in the environment at room temperature or vacuum, thermoelastic damping caused by the micro structure of internal energy dissipation is considered to be the main mode of energy dissipation. So far, micro structure of thermoelastic damping has become the main emphasis of the research.

Zener ${ }^{[1]}$ first introduced the concept of thermoelastic damping. Based on a viscoelastic solid model system, the lateral flexural vibrations of isotropic and homogeneous thermoelastic rectangular beams are investigated. He also put forward the concept of thermalelastic viscoelastic solid in relaxation time. However, A series of approximations are used in the Zener' ${ }^{[1,2]}$ study, the frequency shift of the microstructure due to thermoelastic damping is not obtained. Lifshitz and Rouckes ${ }^{[3]}$ modified Zener's theory and derived exact solutions of thermoelastic damping and expressions for frequency shift. The result shows that the quality factor of microstructure is irregularly distributed with the change of structure size. According to the comparison of the studies of Zener and Lifshitz, there are always damping peaks. Zener's result shows that when beam size

\footnotetext{
a Corresponding author: liuyuting0531@126.com
}

increases it deviates from the exact values of Lifshitz. The study of Lifshitz has attracted more and more scholars' attention to thermoelastic damping and thermoelastic models of different microstructures.

Jagannathan $^{[4]}$ used a single degree of freedom model to analyze the thermal properties of the cantilever beam. Dileesh $\mathrm{V}^{[5]}$ analysed two end clamped beams based on Timoshenko. X.Guo ${ }^{[6]}$ analysed the thermoelastic damping of a two end clamped beam and a cantilever beam with a rectangular hole. The results show that the rectangular hole makes the quality factor of the resonant structure decrease obviously.

In piezoelectric devices, there is also the role of thermoelastic coupling, and the impact on the characteristics of piezoelectric devices, as shown in Figure $1^{[7]}$. Zhou ${ }^{[8]}$ analyzed the temperature and thermal stress distributions of piezoelectric actuators under dynamic loading using analytical methods. It is concluded that the increase of temperature will cause the aging of piezoelectric actuator structure and even the damage of structure. Based on Euler-Bernoulli beam, D Grover ${ }^{[9]}$ established thermoelastic coupling loop expressions of piezoelectric beam transverse vibration mode. The effects of electromotive force, thermal relaxation time, thermoelastic coupling and the size of beam on the energy dissipation caused by thermoelastic damping are analyzed under free boundary conditions. Nourmohammadi ${ }^{[10]}$ established the thermoelastic coupling response spectrum of the piezoelectric composite beam. Qiu and other ${ }^{[11]}$ used analytical method, 
numerical method and experimental verification to analyze the thermoelastic damping of piezoelectric beams of different structures.

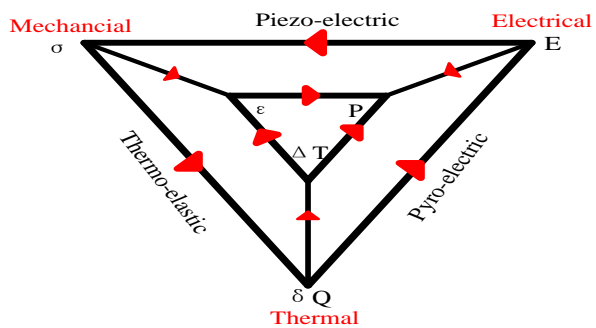

Figure 1. Schematic of Force-electric-thermal coupling model for piezoelectric materials

On the basis of previous work, this paper establishes piezoelectric thermoelastic coupling governing equations under different vibration. By the method of numerical calculation and simulation, the relationship between the piezoelectric output characteristics and the vibration modes is studied, and the effects of piezoelectric thermoelastic coupling on thermoelastic damping and frequency shift are researched, which means the influence of the temperature field on the piezoelectric structure under different vibration modes is obtained. Finally, through experimental methods, the phenomenon that frequency shift caused by piezoelectric thermoelastic coupling is verified.

\section{Theoretical Description of Piezoelectric Thermoelastic Coupling.}

\subsection{Piezoelectric Thermoelastic Coupling under Free Vibration}

As shown in Figure 2, a schematic diagram of the piezoelectric harvester structure, which consisting of a cantilever steel beam, a mass block and piezoelectric material (PZT-5H) is presented. The piezoelectric material is tiled on the cantilever beam structure, and the silver electrode is applied to the upper and lower surfaces of the piezoelectric layer. Assuming that the piezoelectric structure is isotropic. The length, width and thickness of the beam as $\mathrm{x}$ axis, $\mathrm{y}$ axis and $\mathrm{z}$ axis respectively.

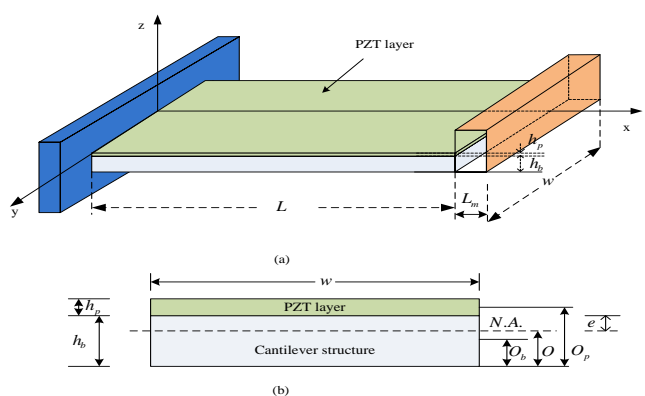

Figure 2. Schematic drawing of piezoelectric structure

Based on the piezoelectric constitutive equations and the heat entropy equation, the piezoelectric thermoelastic constitutive equations are obtained:

$$
\left\{\begin{array}{l}
\varepsilon=\sigma / Y_{E}+\alpha \theta+d_{31} E_{3} \\
D_{3}=d_{31} \sigma+\varepsilon^{T} E_{3}+p \theta \\
S=\rho C \theta / T_{0}+\alpha \varepsilon+p E_{3}
\end{array}\right.
$$

Wher $\varepsilon_{x}, \sigma_{x}, Y_{E}, \alpha, d_{31}, E_{3}, D_{3}, \varepsilon^{T}, p, S, C, \rho$ and $T_{0}$ are strain in $\mathrm{x}$, stress in $\mathrm{x}$, elastic modulus, thermal expansion coefficient, piezoelectric coefficient, electric field, electric displacement, dielectric constant, pyroelectric constant, entropy, heat capacity, density, initial temperature, respectively. Also, $\theta=T-T_{0}$ is temperature variation in which $T$ is defined as temperature field.

The vibration equilibrium equation, the electrical equation and the heat conduction equation are substituted into the Equation(1), the piezoelectric thermoelastic coupling governing equations under free vibration can be obtained as:

$$
\left\{\begin{array}{l}
\rho A \frac{\partial^{2} w}{\partial t^{2}}+\left(Y_{E} I-d_{31} \beta_{2} Y_{E} I\right) \frac{\partial^{4} w}{\partial x^{4}}+\left(Y_{E} \alpha+Y_{E} d_{31} \beta_{1}\right) \frac{\partial^{2} M_{T}}{\partial x^{2}}=0 \\
\left(\varepsilon^{T}-Y_{E} d_{31}^{2}\right) \frac{\partial E_{3}}{\partial z}=Y_{E} d_{31} \frac{\partial^{2} w}{\partial x^{2}}-\left(p-Y_{E} d_{31} \alpha\right) \frac{\partial \theta}{\partial z} \\
\kappa\left(\frac{\partial^{2} \theta}{\partial x^{2}}+\frac{\partial^{2} \theta}{\partial z^{2}}\right)=(1-2 v) \alpha T_{0} \frac{\partial}{\partial t}\left(-z \frac{\partial^{2} w}{\partial x^{2}}\right)+\left(\rho C+2(1+v) \alpha^{2} T_{0}\right) \frac{\partial \theta}{\partial t}+\left(2 \alpha v d_{31}+p\right) T_{0} \frac{\partial E_{3}}{\partial t}
\end{array}\right.
$$

It is necessary to consider the initial and boundary conditions of different physical fields of piezoelectric structures in order to solve the piezoelectric thermoelastic coupling equations. In the mechanical domain, the initial conditions for piezoelectric cantilever structures are as follows; In the temperature domain, it is assumed that there is no heat flux in the upper and lower surfaces of the piezoelectric material; In the electric field, the initial potential is zero. Then the initial condition of piezoelectric structure can be presented as:

$$
\left\{\begin{array}{l}
\left.W\right|_{x=0}=\mathrm{d} W /\left.\mathrm{d} x\right|_{x=0}=0, \mathrm{~d}^{2} W /\left.\mathrm{d} x^{2}\right|_{x=L}=\mathrm{d}^{3} W /\left.\mathrm{d} x^{3}\right|_{x=L}=0 \\
\partial \Theta / \partial z=0 \text { at } z= \pm H_{p} / 2 \\
E_{3} \mid=0
\end{array}\right.
$$

In order to analyze the effect of piezoelectric thermoelastic coupling on the output of piezoelectric structures, it is assumed that the temperature changes, the electric field changes and the displacement are all about the resonant forms of time:

$$
\left\{\begin{array}{l}
w(x, t)=W(x) \exp (i \omega t) \\
E(x, z, t)=\Phi(x, z) \exp (i \omega t) \\
\theta(x, z, t)=\Theta(x, z) \exp (i \omega t)
\end{array}\right.
$$

The upper type is substituted into the thermoelastic coupled equations, the partial differential equations for the displacement, the potential and the temperature can be written as:

$$
\left\{\begin{array}{l}
\left(Y_{E} I-d_{31} \beta_{2} Y_{E} I\right) \frac{\partial^{4} W}{\partial x^{4}}+\left(Y_{E} \alpha+Y_{E} d_{31} \beta_{1}\right) \frac{\partial^{2} M_{\Theta T}}{\partial x^{2}}-\omega^{2} \rho A W=0 \\
\Phi=-\beta_{1} \Theta+\beta_{2} \frac{\partial^{2} W}{\partial x^{2}} \\
\kappa \frac{\partial^{2} \Theta}{\partial x^{2}}+\kappa \frac{\partial^{2} \Theta}{\partial z^{2}}=i \omega\left(\beta_{2}^{*} \Theta-\beta_{1}^{*} z \frac{\partial^{2} W}{\partial x^{2}}\right)
\end{array}\right.
$$


where $M_{\Theta T}=\int_{-h / 2}^{h / 2} b \Theta z d z$

The initial condition of piezoelectric structure is substituted into Equation (5), the displacement solution, temperature solution and potential of the piezoelectric structure can be expressed as:

$$
\left\{\begin{array}{l}
W(x, t)=\sum_{j=1}^{\infty} \hat{A}_{j} W(\mathrm{x}) \mathrm{e}^{i \omega t} \\
\Theta(x, z, t)=\frac{\beta_{1}^{*} \eta^{2}}{\beta_{2}^{*}}\left(z-\frac{\sin k z}{k \cos (k h / 2)}\right) \sum_{j=1}^{\infty} \hat{A}_{j} W_{\Theta}(x) \mathrm{e}^{i \omega t} \\
\Phi(x, t)=\left(\left(\frac{\beta_{1} \beta_{1}^{*}}{\beta_{2}^{*}}\left(z-\frac{\sin k z}{k \cos (k h / 2)}\right)\right)-\beta_{2}\right) \eta^{2} \sum_{j=1}^{\infty} \hat{A}_{j} W_{\Theta}(x) \mathrm{e}^{i \omega t}
\end{array}\right.
$$

where

$$
W_{\Theta}(x)=-\cos (\eta x)-\cosh (\eta x)-\frac{\cos \eta L+\cosh \eta L}{\sin \eta L+\sinh \eta L}(-\sin (\eta x)-\sinh (\eta x)) .
$$

\subsection{Piezoelectric Thermoelastic Coupling under Forced Vibration}

In this section, the thermoelastic coupling characteristics of the piezoelectric structure under forced vibration are analyzed. And the vibration response, temperature variation and voltage output of the piezoelectric structure in the forced vibration are obtained.

The piezoelectric structure is subjected to transverse forced vibration by external loads. The schematic diagram is shown in Figure 2 above. Using the Euler Bernoulli beam hypothesis theory, the force-electrothermal coupled constitutive equation for the forced vibration of piezoelectric structures is also shown in Equation (1).

The transverse forced vibration equilibrium equation of piezoelectric structure can be expressed as:

$$
\rho S \frac{\partial^{2} w}{\partial t^{2}}+\frac{\partial^{2} M}{\partial x^{2}}=f(t)
$$

Where $f(t)$ is the load along thickness direction, $M(x, t)=-\int_{-h / 2}^{h / 2} b \sigma_{x} z d z$

The vibration balance equation can be written as:

$$
\rho S \frac{\partial^{2} w}{\partial t^{2}}+Y_{E} I \frac{\partial^{4} w}{\partial x^{4}}+\frac{\partial^{2} M_{T}}{\partial x^{2}}+\frac{\partial^{2} M_{E}}{\partial x^{2}}=f(t)
$$

The governing equations of piezoelectric structures can be expressed as:

$$
\left\{\begin{array}{l}
Y_{E} I \frac{\partial^{4} w}{\partial x^{4}}+\rho S \frac{\partial^{2} w}{\partial t^{2}}+\frac{\partial^{2} M_{T}}{\partial x^{2}}+\frac{\partial^{2} M_{E}}{\partial x^{2}}=f(t) \\
\left(\varepsilon^{T}-Y_{E} d_{31}^{2}\right) \frac{\partial E_{3}}{\partial z}-Y_{E} d_{31} \frac{\partial^{2} w}{\partial x^{2}}+\left(p-Y_{E} d_{31} \alpha\right) \frac{\partial \theta}{\partial z}=0 \\
\kappa\left(\frac{\partial^{2} \theta}{\partial x^{2}}+\frac{\partial^{2} \theta}{\partial z^{2}}\right)=(1-2 v) \alpha T_{0} \frac{\partial}{\partial t}\left(-z \frac{\partial^{2} w}{\partial x^{2}}\right)+\left(\rho C+2(1+v) \alpha^{2} T_{0}\right) \frac{\partial \theta}{\partial t}+\left(2 \alpha v d_{31}+p\right) T_{0} \frac{\partial E_{3}}{\partial t}
\end{array}\right.
$$

In order to analyze the influence of thermoelastic coupling on the output of piezoelectric structures, it is assumed that the temperature changes, the electric field changes and the displacement are all about the resonant forms of time. The displacement solution, temperature solution and potential of transverse forced vibration of piezoelectric structures can be expressed as:

$$
\left\{\begin{array}{l}
W_{f}(x, t)=\sum_{j=1}^{\infty} \hat{A}_{j} W(\mathrm{x}) \mathrm{e}^{i \omega_{f} t}-\frac{F(t)}{\omega^{2} \rho A} \\
\Theta_{f}(x, z, t)=\frac{\beta_{1}^{*} \eta^{2}}{\beta_{2}^{*}}\left(z-\frac{\sin k z}{k \cos (k h / 2)}\right) \sum_{j=1}^{\infty} \hat{A}_{j} W_{\Theta}(x) \mathrm{e}^{i \omega_{f} t} \\
\Phi_{f}(x, t)=\left(\left(\frac{\beta_{1} \beta_{1}^{*}}{\beta_{2}^{*}}\left(z-\frac{\sin k z}{k \cos (k h / 2)}\right)\right)-\beta_{2}\right) \eta^{2} \sum_{j=1}^{\infty} \hat{A}_{j} W_{\Theta}(x) \mathrm{e}^{i \omega_{f} t}
\end{array}\right.
$$

\subsection{Frequency Shift and Piezoelectric Thermoelastic Damping}

Based on the above analysis, the solution of the physical parameters of piezoelectric structures under piezoelectric thermoelastic coupling is obtained. The internal temperature changes not only affect the vibration response and voltage output, but also cause the resonant frequency of the structure. The structure frequency under piezoelectric thermoelastic coupling can be expressed as:

$$
\begin{aligned}
\omega & =\eta^{2} \sqrt{X / \rho A}=\omega_{0} \sqrt{X_{1}\left(1+X_{2}(1+f(k))\right)} \\
& =\omega_{0} \sqrt{X_{1}}\left(1+X_{2}(1+f(k)) / 2\right)
\end{aligned}
$$$$
\text { where } f(k)=\frac{24}{k^{3} h^{3}}\left(\frac{k H_{p}}{2}-\tan \frac{k H_{p}}{2}\right) \text {. }
$$

Because the temperature gradient along the thickness direction of the piezoelectric structure is much larger than that of the horizontal direction, the frequency can be obtained as:

$$
\begin{aligned}
\omega & =\omega_{0} \sqrt{X_{1}}\left(1+\frac{X_{2}}{2}\left(1-\frac{12}{(1+i) \xi^{3}}\left(\frac{(1-i) \xi}{2}-\tan \frac{(\xi-i \xi)}{2}\right)\right)\right) \\
& =\omega_{0} \sqrt{X_{1}}\left(1+\frac{X_{2}}{2}\left(1-\frac{6}{\xi^{3}}\left(\frac{\sinh \xi-\sin \xi}{\cos \xi+\cosh \xi}+i\left(\frac{\sinh \xi+\sin \xi}{\cos \xi+\cosh \xi}-\frac{\xi}{2}\right)\right)\right)\right)
\end{aligned}
$$

It is known from Equation (12) that the real and imaginary parts of the frequency represent the shift of the resonant frequency and the vibration of the piezoelectric structure under thermoelastic coupling, respectively. The real and imaginary parts respectively can be written as:

$$
\begin{aligned}
& \operatorname{Re}(\omega)=\omega_{0} \sqrt{X_{1}}\left(1+\frac{X_{2}}{2}\left(1-\frac{6}{\xi^{3}} \frac{\sinh \xi-\sin \xi}{\cos \xi+\cosh \xi}\right)\right) \\
& \operatorname{Im}(\omega)=\frac{\omega_{0} X_{2} \sqrt{X_{1}}}{2}\left(\frac{6}{\xi^{3}} \frac{\sinh \xi+\sin \xi}{\cos \xi+\cosh \xi}-\frac{6}{\xi^{2}}\right)
\end{aligned}
$$

The resonant frequency drift of the piezoelectric structure caused by the piezoelectric thermoelastic coupling effect is defined as $\omega_{S}$ :

$\omega_{S}=\left|\frac{\operatorname{Re}(\omega)-\omega_{0}}{\omega_{0}}\right|=\left|\sqrt{X_{1}}-1+\frac{\sqrt{X_{1}} X_{2}}{2}\left(1-\frac{6}{\xi^{3}} \frac{\sinh \xi-\sin \xi}{\cos \xi+\cosh \xi}\right)\right|$

The amount of energy dissipation in the vibration of piezoelectric structures is piezoelectric thermoelastic damping, which is the reciprocal of the structure quality factor.The thermoelastic damping can be expressed as: 


$$
Q^{-1}=2\left|\frac{\operatorname{Im}(\omega)}{\operatorname{Re}(\omega)}\right|
$$

\section{Simulation Results}

For piezoelectric structures, when coupled with temperature field, the output is affected by piezoelectric thermoelastic coupling. In order to analyze the influence of temperature on the output characteristics of piezoelectric structure under piezoelectric thermoelastic coupling, the dynamic responses of structures under different physical field coupling mechanisms are simulated and analyzed. The structural dimensions and material property parameters in the simulation analysis are shown in Tables 1 and 2.

Table 1. Structure and material parameters of piezoelectric composite beam

\begin{tabular}{|c|c|c|c|}
\hline Structure & Cantilever & Mass & Piezoelectric layer \\
\hline $\mathbf{E} / \mathbf{G p a}$ & 200 & 200 & 60 \\
\hline $\boldsymbol{\rho} / \mathbf{K g} / \mathbf{m}^{\mathbf{3}}$ & 7850 & 7850 & 7700 \\
\hline $\boldsymbol{U}$ & 0.3 & 0.3 & 0.28 \\
\hline $\mathbf{E} / \mathbf{F} / \mathbf{m}$ & & & 1700 \\
\hline $\mathbf{d} \mathbf{3 1} / \mathbf{C} / \mathbf{N}$ & & & $-273 \times 10^{-12}$ \\
\hline length/mm & 28 & 10 & 28 \\
\hline Width/mm & 25 & 25 & 25 \\
\hline High/mm & 0.2 & 4.8 & 0.2 \\
\hline
\end{tabular}

Table 2. Geometrical and physical parameters of piezoelectric materials

\begin{tabular}{|c|c|c|}
\hline Structure & substrate/steel & $\begin{array}{c}\text { Piezoelectric } \\
\text { layer/PZT-5H }\end{array}$ \\
\hline $\boldsymbol{\alpha} / \mathbf{1} / \mathbf{K}$ & $12 \times 10^{-6}$ & $4.7 \times 10^{-6}$ \\
\hline $\mathbf{K} / \mathbf{W m}^{-1} \mathbf{K}^{-1}$ & 45 & 1.5 \\
\hline $\mathbf{C p} / \mathbf{J K g}^{-1} \mathbf{K}^{-\mathbf{1}}$ & 460 & 420 \\
\hline $\boldsymbol{p} / \mathbf{C K}^{-1} \mathbf{m}^{-\mathbf{2}}$ & & $-452 \times 10^{-6}$ \\
\hline $\mathbf{T}_{\mathbf{0}} / \mathbf{K}$ & 300 & 300 \\
\hline
\end{tabular}

\subsection{Simulation of Piezoelectric Thermoelastic Coupling under Free Vibration}

The free vibration response at the first two natural frequencies of the structure is shown in Figure 3 (a) (f). According to the simulation results, the output response and the temperature are the highest at the first-order resonance, and the vibrational mechanical energy becomes larger, that is, the force-electric-thermal coupling strength is the strongest. The maximum response of thermoelastic first-order vibration is $4.83 \mathrm{~mm}$, the highest temperature gradient is $8.04 \mathrm{~K}$ at the fixed end of the structure, and the response voltage output is $214 \mathrm{v}$.
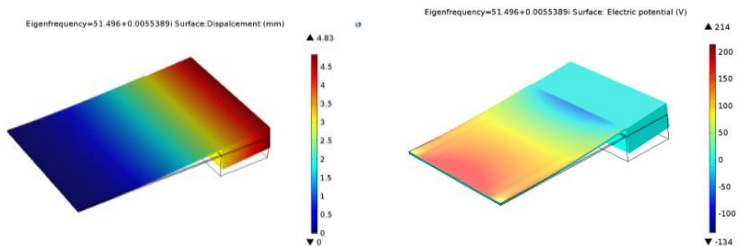

(a) The first order of the vibration displacement distribution

(b) The first order of the voltage distribution
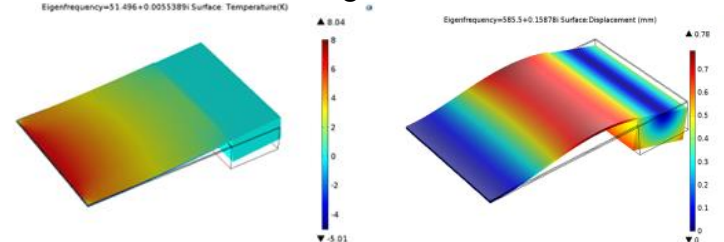

(c) The first order of the temperature distribution

(d) The second order of the vibration displacement distribution
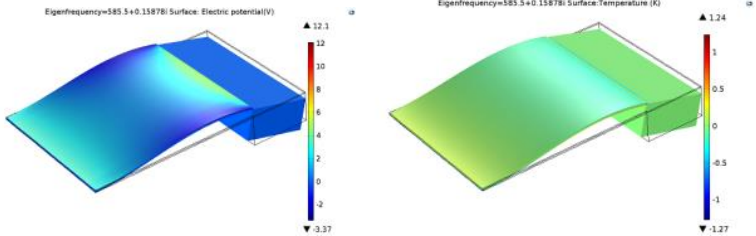

(e) The second order of the voltage distribution

(f) The second order of the temperature distribution

Figure 3. Simulation results of the first two order free vibrations of piezoelectric thermoelastic coupling

The structural output characteristics of piezoelectric layer, contact layer (interlayer) and structural layer path are analyzed. The output responses of the different layers are shown in Figure 4 (a) (f). The results show that the temperature response in the piezoelectric layer is slightly lower than that in the substrate structure. Moreover, the voltage response at the first-order resonant frequency is stronger than that at the two-order free vibration in the piezoelectric layer, and the same phenomenon exists for the temperature distribution. In the contact layer, due to the difference of thermal expansion coefficient between the piezoelectric layer and the substrate layer, the vibration response characteristics of the contact layer are different. In the same vibration, the temperature distribution of the contact layer is larger than that of the piezoelectric layer, and the mechanical energy appears more attenuation through the contact layer. The temperature distribution at the fixed end is analyzed by thickness direction and different layer paths, the result is shown in Figure 4 (e), (f). According to the result, when the fixed end in the structure is vibrating near the resonant frequency, the lower layer is in the tensile state, and the piezoelectric layer is in the compression state, so that the temperature increases with the increase of the thickness of the structure, the temperature is higher in the first mode vibration. Meanwhile, the temperature change 
of the contact layer is greater than that of the piezoelectric layer.
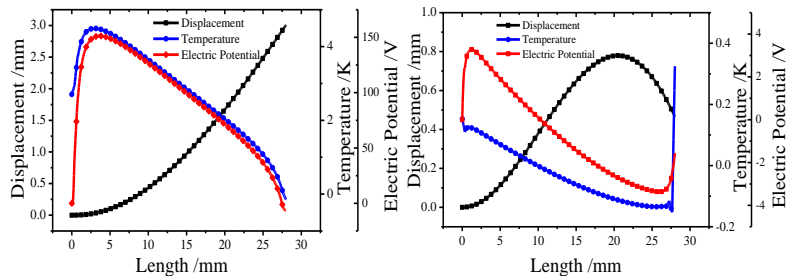

(a) Temperature, voltage and displacement distribution in the first mode of piezoelectric layer

(b) Temperature, voltage and displacement distribution in the second mode of piezoelectric layer
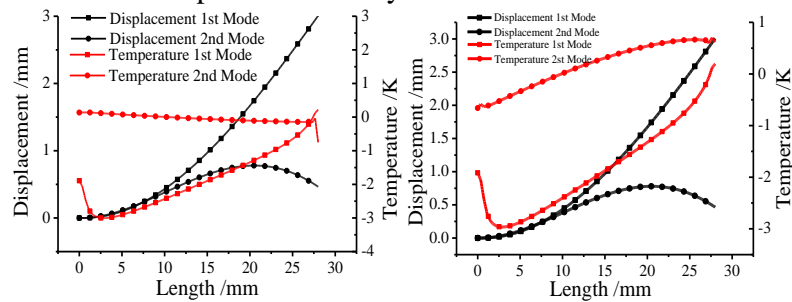

(c) Temperature and displacement distribution in the first two modes of contact layer

(d) Temperature and displacement distribution in the first two modes of substrate layer
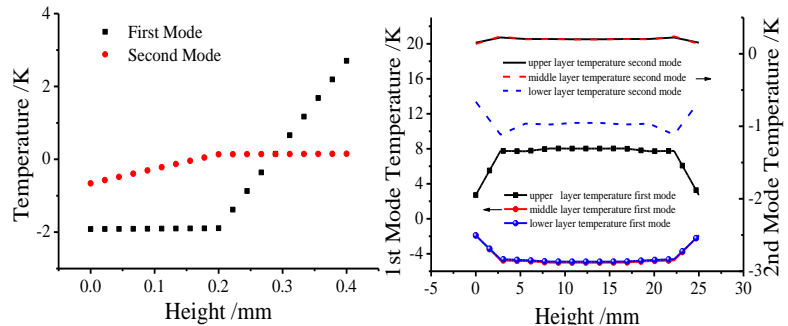

(e) The first two order temperature distributions in the $\mathrm{Z}$ direction at the fixed end

(f) The temperature distribution of the structure layer in the $\mathrm{Y}$ direction at fixed end

Figure 4. Analysis results of free vibration paths of piezoelectric thermoelastic coupling

\subsection{Simulation of Piezoelectric Thermoelastic Coupling under Forced Vibration}

The external excitation is applied to the piezoelectric structure, the external acceleration is $9 \mathrm{~m} / \mathrm{s}^{2}$, and the excitation frequency is close to the natural frequency of the structure. The response of the forced vibration of piezoelectric thermoelastic coupling are shown in Figure 5 (a) (f). According to the simulation results, the output characteristic of piezoelectric structure under forced vibration is larger than that output under free vibration. In the forced vibration, the maximum response of thermoelastic first-order vibration is $4.99 \mathrm{~mm}$, the highest temperature gradient is $8.18 \mathrm{~K}$ at the fixed end in the structure, and the response voltage output is $216 \mathrm{v}$. Similarly, under the forced vibration, the output response of the structure under the first-order forced vibration is maximum.
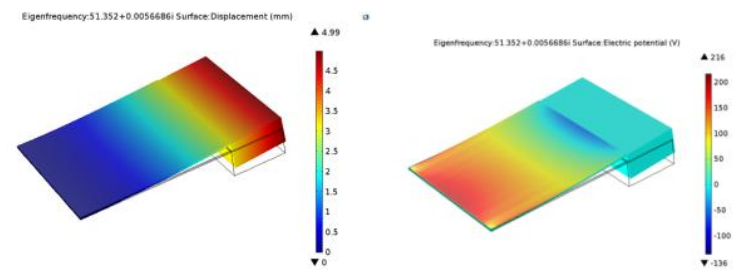

(a) The first order of the vibration displacement distribution,

(b) The first order of the voltage distribution
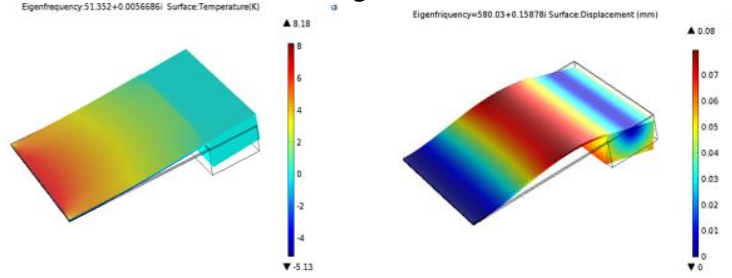

(c) The first order of the temperature distribution

(d) The second order of the vibration displacement distribution
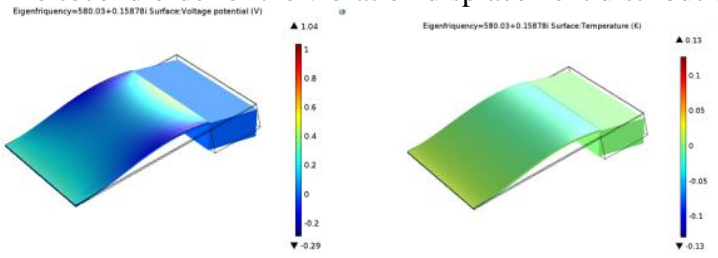

(e) The second order of the voltage distribution

(f) The second order of the temperature distribution

Figure 5. Simulation results of the first two order forced vibration of piezoelectric thermoelastic coupling

The output responses of the different layers under forced vibration are shown in Figure 6 (a) (f). As a result, the temperature response in the piezoelectric layer is slightly lower than that in the substrate structure due to the material properties; Moreover, the voltage response at the two-order resonant frequency in the piezoelectric layer is lower than that at the first-order forced vibration, and the temperature distribution is similar. The vibration response, voltage output and dissipation energy of piezoelectric structures under forced vibration are higher than that under free vibration.
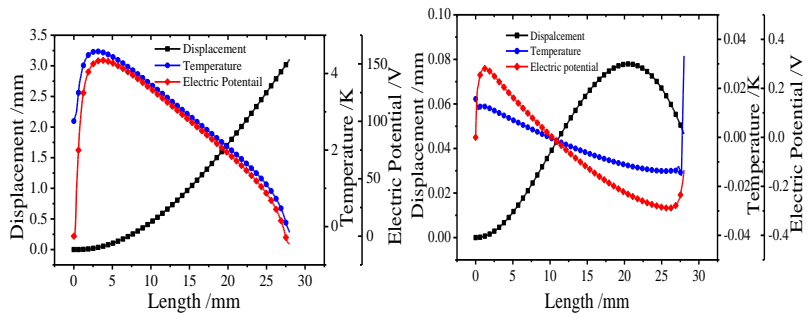

(a) Temperature, voltage and displacement distribution in the first mode of piezoelectric layer

(b) Temperature, voltage and displacement distribution in the second mode of piezoelectric layer

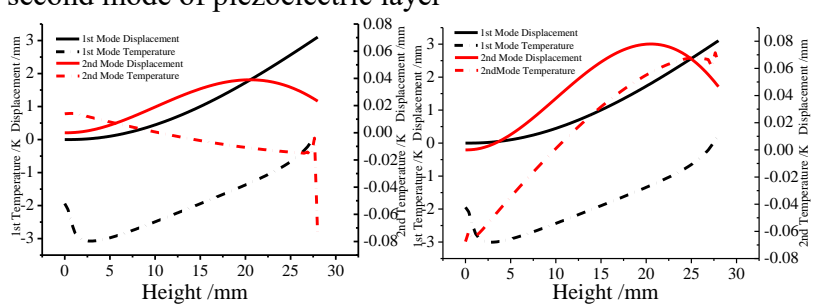

(c) Temperature and displacement distribution in the first two modes of contact layer 
(d) Temperature and displacement distribution in the first two modes of substrate layer
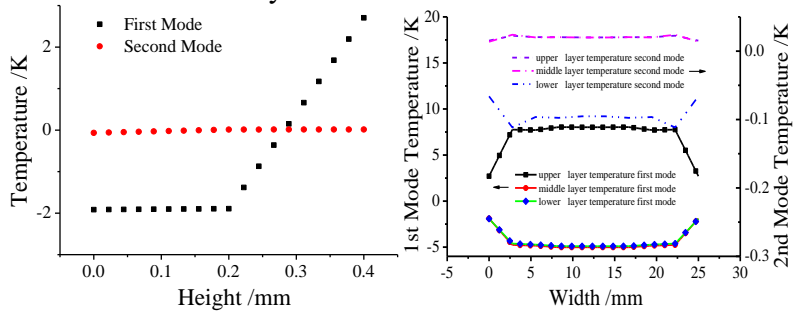

(e) The first two order temperature distribution in the $\mathrm{Z}$ direction at the fixed end

(f) The temperature distribution of the structure layer in the $\mathrm{Y}$ direction at fixed end

Figure 6. Analysis results of forced vibration paths of piezoelectric thermoelastic coupling

\subsection{Frequency Shift and Piezoelectric Thermoelastic Damping}

The relationship between the frequency shift ratio and the structure thickness is shown in Figure 7. It is known from the figure that the frequency shift ratio rises rapidly with the increase of the piezoelectric layer thickness, and gradually reaches a certain value when it reaches a certain size. When the vibration attenuation reaches the maximum, it is the critical thickness. At the critical thickness, the structure frequency shift ratio changes most acutely. According to the simulation results, the frequency shift caused by the piezoelectric elastic coupling effect is near $3.8 \mathrm{e}-4$. Comparing the numerical results and drawing the difference curves, two calculations are basically consistent.

The simulation results of piezoelectric thermoelastic damping are shown in Figure 8. According to the simulation results, the thermoelastic damping increases when the piezoelectric layer thickness is increased, after reaching the peak, it gradually gose down by futher increse the layer thinkness. The peak value of thermoelastic damping is $7 \mathrm{e}-4$, at this time the thickness of the structure is the same as the numerical results. The simulation results of thermoelastic damping have the same trend with the numerical results, but only slightly different at the peak.

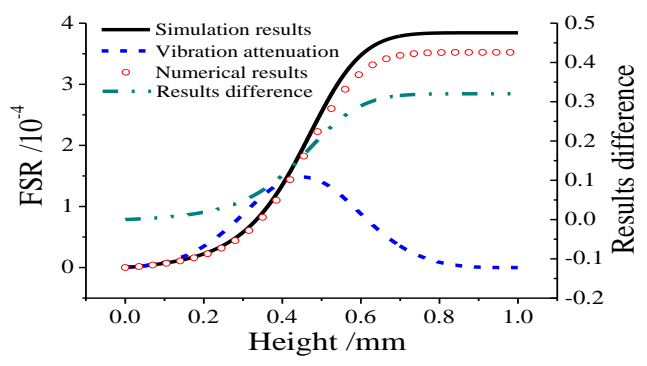

Figure 7.The curve of free vibration frequency shift ratio and vibration attenuation

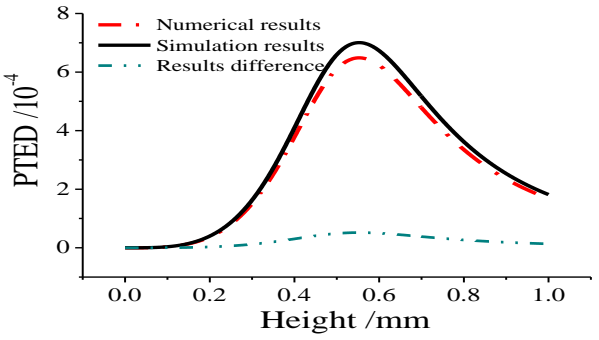

Figure 8. Free vibration piezoelectric thermoelastic damping varies with piezoelectric layer thickness

The relationship between the frequency shift ratio and the structure thickness under forced vibration is shown in Figure 9. Form the comparison of Figure 7 with Figure 9, of Figure 8 with Figure 10, it is obvious that the change curve under forced vibration is consistent with the curve under free vibration. But the frequency shift ratio is stable near $3.9 \mathrm{e}-4$, the peak value of the thermoelastic damping is $7.1 \mathrm{e}-4$. In contrast to the results of the free vibration, the frequency shift is greater under forced vibration.

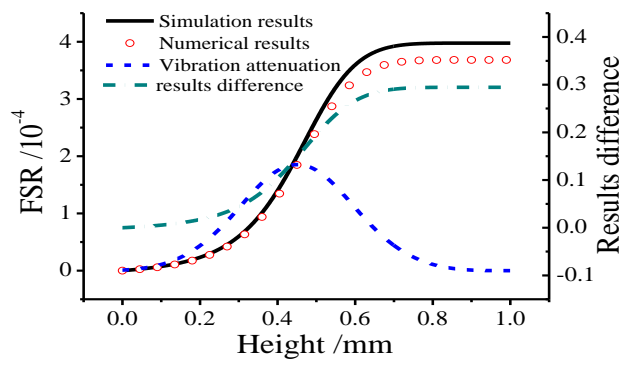

Figure 9. The curve of forced vibration frequency shift ratio and vibration attenuation

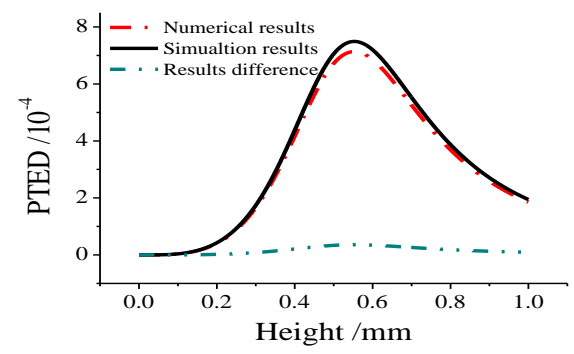

Figure 10. Forced vibration piezoelectric thermoelastic damping varies with piezoelectric layer thickness

The natural frequency of the forced vibration is lower than the natural frequency of the free vibration. This is because the structure deformation at the forced vibration is greater than that at the free vibration, the temperature gradient is higher, the more influence on the structural stiffness, and further reduces the natural frequency.

\section{Experiments}

From the above analysis, it is known that the thermoelastic coupling characteristics of the piezoelectric structure will affect the resonant frequency of the structure, resulting in the frequency shift of the structure. In this section, the thermoelastic coupling characteristics of piezoelectric structures are analyzed by experimental 
tests. The design and fabrication of the piezoelectric structure and experimental installation are shown in Figure 11. In test, the output excitation signal of the wave generator (RIGOL, DG2041A) through the power amplifier to vibration table drives the harvester, the acceleration sensor (Model, 393B04) tests the acceleration signal, the dynamic signal analyzer (COCO80 ) records the acceleration signal, the output voltage of open circuit and access load of piezoelectric energy harvesting device is recorded by oscillograph. Among them, the structural parameters and material properties of piezoelectric structures are shown in Tables 1 and 2 .

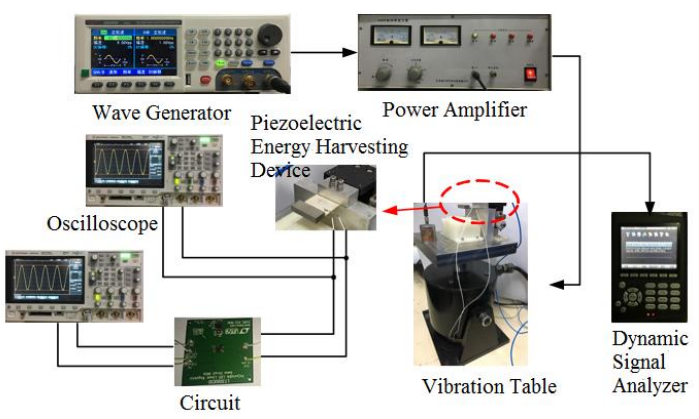

Figure 11. Schematic diagram of the experimental setup

Under the external acceleration excitation, the open circuit voltage of the piezoelectric harvester is shown in Figure 12. It is shown that when the external excitation is consistent with the structure resonant frequency, the output voltage reaches its peak, and its resonant frequency is near $90 \mathrm{~Hz}$. However, as the experiment continues, the frequency corresponding to the peak output is inconsistent, which means that the structural resonance frequency shifts during the vibration process.

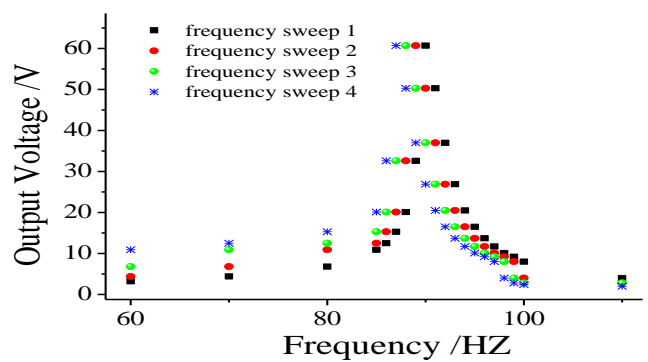

Figure 12 Experimental results of the piezoelectric harvester

Continuous testing of the piezoelectric structure, the resonance frequency shift as shown in Figure 13. From the result, it is found that when the piezoelectric material is fixed, the frequency shift ratio decreases with the increase of vibration time, and eventually stabled at 6e-5. According to the theoretical analysis, when the piezoelectric material layer is $0.2 \mathrm{~mm}$, the thermal elastic frequency ratio shifts its original natural frequency by $4 \mathrm{e}-5$. Therefore, it can be concluded that the structural frequency shift is mainly caused by thermoelastic dissipation during the experiment, and the correctness of the theory and simulation is verified.

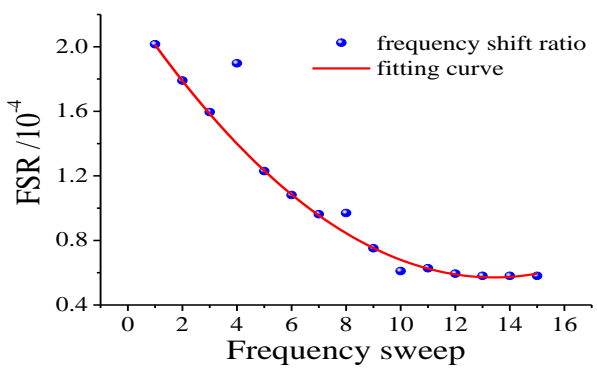

Figure 13. Frequency shift ratio of the structure during experiments

\section{Conclusions}

Aimed at the effect of piezoelectric thermoelastic coupling on both free and forced vibrations, this paper established the piezoelectric thermoelastic coupling governing equations. By introducing the thermal concept to the piezoelectric structure, the displacement distribution, the voltage distribution, the temperature distributions, thermoelastic damping and frequency shift were derived. Undering free bibration or forced bibration, the piezoelectric thermoelastic damping increases when the piezoelectric layer thickness is increased. When reaching the peak value, it gradually gose down by futher increse the layer thinkness. The piezoelectric frequency shift increases rapidly with the increase of the piezoelectric layer thickness, and gradually reaches a certain value when it reaches a certain size. On the other hand, the piezoelectric vibration leads to the vibration attenuation of the piezoelectric structure. The vibration attenuation is the maximum at the critical thickness. The frequency shift varies widely at the critical thickness. Therefore, when analyzing the multi-field coupling of piezoelectric structures, it needs to consider the physical quantities of the piezoelectric structures in different physical field coupling. The optimum piezoelectric structure size is designed to avoid the maximum thermoelastic damping, and the structure achieves the maximum quality factor and the highest voltage output.

\section{References}

1. Zener C. Phys. Rev. 52, 230 (1937)

2. Zener C. Phys. Rev. 53, 90 (1938)

3. Lifshitz R. , Roukes M.L. Phys. Rev. B. 61, 5600 (1999)

4. Rajagopalan J. , Saif, M.T.A. J APPL MECH. 74, 461 ( 2007)

5. Parayil D.V., Kulkarni S.S. , Pawaskar D.N. INT J MECH SCI. 94, 10 (2015)

6. Guo X. , Yi Y.B., Pourkamali S. INT J MECH SCI. 74, 73 (2013)

7. Ahmad S.N. , Upadhyay C.S. , Venkatesan C. SMART MATER STRUCT. 15, 401 (2006)

8. Zhou S.W. , Rogers C.A. J INTEL MAT SYST STR. 6, $372(1995)$

9. Grover D. , Sharma J.N. J INTEL MAT SYST STR. 23, 77 (2012) 
10. Nourmohammadi Z., Prabhakar S., Vengallatore S.

J MICROELECTROMECH S. 22, 747 (2013)

11. Qiu H. , Ababneh A. , Feili D. MICROSYST TECHNOL. 22, 2017 (2016) 